Research Article

\title{
Trends in Opioid Use in a Cohort of Patients with Rheumatoid Arthritis
}

\author{
Manuel E. Machado-Duque (D), ${ }^{1,2}$ Diana Marcela Ramírez-Valencia, ${ }^{1}$ \\ María Mónica Murillo-Muñoz,, ${ }^{1,2}$ and Jorge E. Machado-Alba $\mathbb{D D}^{1}$ \\ ${ }^{1}$ Grupo de Investigación en Farmacoepidemiología y Farmacovigilancia, Universidad Tecnológica de Pereira-Audifarma S.A, \\ Pereira, Colombia \\ ${ }^{2}$ Grupo Biomedicina, Fundación Universitaria Autónoma de Las Américas, Pereira, Colombia
}

Correspondence should be addressed to Jorge E. Machado-Alba; machado@utp.edu.co

Received 21 February 2020; Accepted 26 June 2020; Published 17 July 2020

Academic Editor: Manfred Harth

Copyright (c) 2020 Manuel E. Machado-Duque et al. This is an open access article distributed under the Creative Commons Attribution License, which permits unrestricted use, distribution, and reproduction in any medium, provided the original work is properly cited.

\begin{abstract}
The objective was to determine the trend in the use of opioid analgesics in a cohort of patients diagnosed with and treated for rheumatoid arthritis (RA) in 24 cities in Colombia. This retrospective cohort study included adult patients diagnosed with RA, which was managed in a specialized institution in Colombia between January 2011 and December 2012. The first rheumatology visit was recorded as an index date, and monthly monitoring of the analgesic medication received was performed until December 2017. Sociodemographic variables, the use of opioids, and concomitant prescriptions were evaluated. A total of 1,329 patients diagnosed with and treated for RA were included; they had a mean age of $61.2 \pm 11.8$ years and were predominantly females $(n=936 ; 82.9 \%)$. A total of 1,129 (84.9\%) subjects used opioids for at least one month, and a growing trend, from $13.5 \%$ to $21.4 \%$, was observed in patients who received opioids every month throughout a 7 -year follow-up of the cohort. In total, $46.7 \%$ of the cases used opioids for more than 12 months. The most commonly used opioids were codeine (76.3\%) and tramadol (71.1\%). All patients received conventional disease-modifying antirheumatic drugs (DMARDs), 85.6\% received systemic corticosteroids, $73.9 \%$ received nonsteroidal anti-inflammatory drugs, and $15.9 \%$ received biological DMARDs. A high proportion of opioid use was shown for pain management in patients with RA, in many cases for more than 12 months, in whom the efficacy and especially safety, related to the risk of dependence, should be monitored.
\end{abstract}

\section{Introduction}

Currently, rheumatoid arthritis (RA) affects the adult population, with an estimated prevalence between $0.5 \%$ and $1 \%$ and an incidence of 5-50 per 100,000 population, mainly affecting women over 65 years. RA is a chronic, progressive autoimmune disease characterized by persistent synovial and systemic inflammation associated with joint destruction, whose main symptom is chronic joint pain, which is the most common reason for consultation $[1,2]$.

For the treatment of pain caused by the RA activity, there are multiple classes of drugs, such as opioids. Although the efficacy and relative safety of these drugs have been previously established for short-term use, there is limited evidence regarding their safety upon chronic use [3, 4]. In recent decades, a significant increase in opioid use has become a concern in Europe, Canada, and the United States $[5,6]$, in particular for their narrow therapeutic margin, tolerance, dependence, and adverse events such as delirium, somnolence, and falls [7-9].

People affected by RA pain, along with patients with neoplasm-related pain, have traditionally been treated with opioids. However, recent studies have shown an increase in opioid use, mainly in the first group [10,11]. In addition, the latter group presents an increased risk of long-term use, situation that can lead to related problems such as dependency, addiction, and overdose, as well as an increased risk of death in this population; therefore, the need to rationalize 
the use for short periods and in patients with indications where the benefit outweighs the risk associated with opioids has been identified [10].

The Colombian Health System offers universal coverage through two enrollment regimes, one paid for the employer and employee and another subsidized by the state, which has a list of drugs included in a benefit plan for all patients and containing different analgesics, antirheumatics, and corticosteroids. Given the risks and adverse events that may arise from their chronic use, it was proposed to determine the trend in the use of opioid analgesics in a cohort of patients diagnosed with and treated for RA in 24 cities in Colombia.

\section{Materials and Methods}

An observational retrospective cohort study was conducted, which included all patients with diagnosis of RA, according to the codes of the International Classification of Diseases version 10.0 (ICD-10), and older than 18 years treated with conventional or biological DMARDs (access to DMARDs in Colombia is fully covered by the Health System) at the Specialized Healthcare Provider Institution (Institución Prestadora de Servicios Especializada, IPS-E) in 24 cities in Colombia (Ithe rheumatologists of IPS Especializada, use EULAR criteria for the diagnosis of RA), who were affiliated with seven different insurers or EPS (Entidades Promotoras de Salud). Patients who received RA care between January 2011 and December 2012 were included, and the index date for the start of follow-up was defined as the date of the first specialized consultation within the observation period (the use of medications of interest was the follow-up month by month). Medications dispensed until December 2017 were identified, and patients with a follow-up of less than 6 months and those who were prescribed opioids in the month prior to the index date were excluded.

For the included cases, the medications dispensed by Audifarma, which is the logistics operator in charge of medication delivery to these patients, were determined from its database of drug use by more than 6.5 million people, a database that is widely used in pharmacoepidemiological studies.

A database with the information on each subject was created, which was reviewed by two of the authors and validated by another one and included the following variables:

(1) Sociodemographic and clinical: age, sex, city of residence, EPS insurer, and diagnosis according to ICD-10.

(2) Use of opioids: prescribed drugs: opioid medication classified by international name and partial or total agonist capacity (weak opioids: codeine, dihydrocodeine, hydrocodone, tramadol, and tapentadol; strong opioids: fentanyl, morphine, oxycodone, hydromorphone, and methadone), date of prescription, dose, duration of use of the drug (in months), change in therapy, increased dose, and use of combined opioids. The use of opioids was defined as a prescription of any opioid during the follow-up period. Chronic use was defined as a prescription for 60 or more days at usual doses, and prolonged use was categorized as a continuous prescription for more than 1 year.

(3) Coprescriptions: combined prescriptions drugs of the following groups of medications were identified as follows (on the index date): (a) conventional disease-modifying antirheumatic drugs (DMARDs), (b) biological DMARDs, (c) nonsteroidal anti-inflammatory drugs (NSAIDs), (d) acetaminophen, (e) dipyrone, (f) systemic corticosteroids, (g) antidepressants (tricyclic, selective serotonin reuptake inhibitors (SSRIs), dual-action, and atypical), (h) antiepileptic drugs, and (i) antipsychotics.

The protocol was approved by the Bioethics Committee of the Universidad Tecnológica de Pereira in the category of "research without risk" and waived the authors from obtaining informed consent; the principles of confidentiality established by the Declaration of Helsinki were respected, and in no case was the personal data of the patients considered. This is not an experimental study, and there was no intervention on patients; so, it was not necessary to obtain informed consent according to Resolution ${ }^{\#} 8430$ of 1993 of the Ministry of Health of Colombia.

2.1. Analysis Plan. The database was created in Microsoft Excel 2016 for Windows. Analyses were performed using the statistical package SPSS 24.0 (IBM, New York, NY, USA). The frequencies and proportions of categorical variables and measures of central tendency and dispersion of quantitative variables were determined by univariate analysis according to their normality (Kolmogorov-Smirnov test). Bivariate analyses were performed using the $\chi^{2}$. A model to adjust was used with binary logistic regression to identify variables that were associated with the prescription of opioids, including those variables with plausibility to explain the outcome and those with statistically significant differences $(p<0.05)$ in the bivariate analysis in the dataset with the use of opioids for more than 12 months. A $p$ value $<0.05$ was established as statistically significant.

\section{Results}

From an original cohort of 1,911 subjects with RA who were followed, 1,329 patients who were diagnosed with RA and treated with conventional or biological DMARDs were identified and followed-up at IPS-E during the period of initial observation. The rest were excluded because their follow-up started after the observation period or was less than 6 months. A total of 1,129 (84.9\%) patients used opioids at least once since prescribed as a therapy at any time during the follow-up (mean: 85.7 months; range: 66-90 months); these patients had a mean age of $61.2 \pm 11.8$ years and were predominantly women $(n=936 ; 82.9 \%)$ (Table 1$)$. The patients were from 24 different cities, most frequently from Bogotá $(n=773 ; 68.5 \%)$, Pereira $(n=95 ; 8.4 \%)$, Manizales $(n=84 ; 7.4 \%)$, Medellin $(n=58 ; 5.1 \%)$, and Cali $(n=30$; $2.7 \%)$. 
TABLE 1: Sociodemographic characteristics and use of opioids by group in a cohort of 1,329 patients with rheumatoid arthritis in Colombia.

\begin{tabular}{lcccc}
\hline Variable & $\begin{array}{c}\text { Total patients } \\
(n=1329), \text { number }(\%)\end{array}$ & $\begin{array}{c}\text { Opioids use patients } \\
(n=1129), \text { number }(\%)\end{array}$ & $\begin{array}{c}\text { Opioids use }<12 \text { months } \\
(n=601), \text { number }(\%)\end{array}$ & $\begin{array}{c}\text { Opioids use } \geq 12 \text { months } \\
(n=528), \text { number }(\%)\end{array}$ \\
\hline Age $($ mean, (SD)) & $61.8 \pm 12.1$ & $61.2 \pm 11.8$ & $59.9 \pm 12.3$ & $62.6 \pm 11.0$ \\
$\quad$ Age $<45$ years & $114(8.6)$ & $107(9.5)$ & $74(12.3)$ & $33(6.3)$ \\
$\quad$ Age 45-64.9 & $676(50.9)$ & $602(53.3)$ & $322(53.6)$ & $280(53.0)$ \\
$\quad$ years & $539(40.5)$ & $420(37.2)$ & $205(34.1)$ & $215(40.7)$ \\
$\quad$ Age $\geq 65$ years & $1103(83.0)$ & $936(82.9)$ & $495(82.3)$ & $441(83.5)$ \\
Female & $1102(82.9)$ & $1102(97.6)$ & $584(97.2)$ & $519(98.3)$ \\
Weak opioids $^{a}$ & $167(12.6)$ & $167(14.8)$ & $60(10.0)$ & $107(20.3)$ \\
Strong opioids $^{b}$ & &
\end{tabular}

${ }^{a}$ Weak opioids: codeine, dihydrocodeine, hydrocodone, tramadol, and tapentadol. ${ }^{b}$ Strong opioids: fentanyl, morphine, oxycodone, hydromorphone, and methadone.

On an average in the follow-up, $21.2 \%$ of all patients had a prescription of opioid analgesics every month, with the proportion ranging from $13.5 \%(n=179)$ in January 2011 to $21.4 \%(n=282)$ at the end of December 2017. Figure 1 shows the trend in the use of opioids. The mean duration of the first opioid prescription was $38.1 \pm 21.5$ days (range: $21-327$ ). In total, $46.7 \%(n=528)$ of the patients used opioids for more than 12 months during the observation period (opioid use did not have to be continuous during follow-up).

We identified 416 patients (36.8\%) who used a single opioid analgesic during the observation period, 434 (38.4\%) who used two different opioids, 229 (20.3\%) with three opioids, and 43 (3.8\%) who received up to four different opioids. The most used opioids were codeine and tramadol; Table 2 shows the prescription patterns for the opioid analgesics used.

Regarding the management of RA, it was found that all patients were managed with conventional DMARDs and 179 (15.9\%) with biological DMARDs. In addition, 966 patients (85.6\%) used systemic corticosteroids, and 834 (73.9\%) received nonsteroidal anti-inflammatory drugs (NSAIDs). Furthermore, the use of other concomitant medications, such as acetaminophen $(n=882 ; 78.1 \%)$, antidepressants $(n=412 ; 36.5 \%)$, anticonvulsants $(n=126 ; 11.2 \%)$, benzodiazepines $(n=42 ; 3.7 \%)$, and antipsychotics $(n=12 ; 1.1 \%)$, was observed.

3.1. Multivariate Analysis. The multivariate analysis indicated that patients older than 45 years and those who received antidepressants, antiepileptic drugs, benzodiazepines, biological DMARDs, and systemic corticosteroids were more likely to receive opioid analgesics for a period longer than 12 months, while no variable was found that would reduce this probability (see Table 3 ).

\section{Discussion}

Opioids have been studied in the coadjuvant management of pain in multiple pathologies, including RA. However, their benefits are unknown upon chronic use because of limited evidence regarding the opioid safety and efficacy in these cases $[4,12]$. In this study, a growing trend could be observed in the use of opioids by patients with RA in Colombia. In addition, almost half of the patients received opioids for more than a year, a situation that highlights the risks associated with this therapy, especially in the long-term, and is considered epidemic even in developed countries [13-15].

A study by Lee et al. has shown that the use of opioid analgesics by RA patients doubled in the Consortium of Rheumatology Researchers of North America (CORRONA) cohort in the United States, having increased from $7.4 \%$ in 2002 to $16.9 \%$ in 2015 [16]. A study by Zamora-Legoff et al., also in the United States, which reviewed the chronic use of this group of drugs in patients with RA, found after 10 years of follow-up that $40 \%$ of patients was prescribed with an opioid at least once [10]. Meanwhile, it was found in our study, with an average follow-up of 7 years, that $84 \%$ of the subjects received opioids at least once, probably because of less restriction in their use in this cohort.

The same study by Zamora-Legoff et al. found that $12.0 \%$ of patients with RA received opioid analgesics for more than 60 days, which was considered a chronic use, while in this study, $87.6 \%$ of the subjects received opioids for the same period of time, and $46.7 \%$ used the drugs for more than a year [10], a worrisome situation considering the lack of evidence on opioid efficacy and long-term safety. In fact, there is no published controlled clinical trial that has studied the use of opioids in patients with RA for more than 8 weeks. Although the results of these short-term studies have shown better outcomes than those observed with placebo in terms of the analgesic efficacy, a higher incidence of adverse events has been reported $[4,17]$. For this reason, most clinical practice guidelines recognize the problem of the long-term use of opioids, as well as the lack of clinical evidence and the need to limit the use and potential risks of opioids, posing limitations on the doses used, advising on dose titration precautions, and promoting awareness on the dangers associated with the use of fentanyl patches and methadone [18].

There is a lack of published information about chronic noncancer pain in Colombia. This supports the thesis carried out by Garcia et al. where they propose an undertreatment of chronic pain in Latin America [19].

Ruiz-Iban et al. also described insufficient pain treatment for chronic pain in osteoarthritis in Latin America, proposing an underuse of strong opioid analgesics [20]. Thus, the results of this study serve as a baseline regarding the use of opioids in patients with RA. 


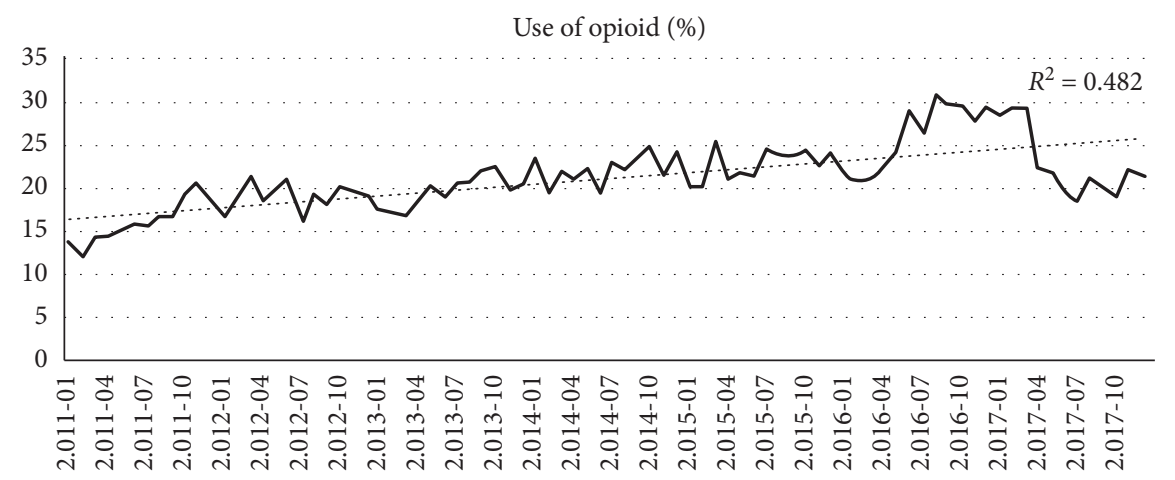

FIgURE 1: Trend in opioid use in a cohort of 1,329 patients with rheumatoid arthritis in Colombia.

TAble 2: Patterns of the use of opioids for pain management in a cohort of 1,129 patients with rheumatoid arthritis in Colombia.

\begin{tabular}{|c|c|c|c|c|c|c|c|c|}
\hline Drug & $n=1129$ & $(\%)$ & $\begin{array}{l}\text { Doses } \\
\text { (mean) }\end{array}$ & $\begin{array}{c}\text { Interval } \\
\text { (no. of doses/day) }\end{array}$ & $\mathrm{nDDD}^{a}$ & $\begin{array}{l}\text { Therapy duration } \\
\text { (months) }\end{array}$ & $\begin{array}{l}\text { Percent } \\
\text { female }\end{array}$ & $\begin{array}{c}\text { Mean age } \\
\text { (years) }\end{array}$ \\
\hline \multicolumn{9}{|l|}{ Weak opioids } \\
\hline Codeine & 861 & 76.3 & 47.7 & 3 & 0.47 & 24.1 & 0.83 & $61.5+11.6$ \\
\hline Tramadol & 803 & 71.1 & $\mathrm{NA}^{*}$ & $\mathrm{NA}^{*}$ & NA & 21.6 & 0.83 & $61.5+11.6$ \\
\hline Hydrocodone & 252 & 22.3 & 12.9 & 3 & 0.86 & 35.6 & 0.85 & $64.9+11.2$ \\
\hline Tapentadol & 9 & 0.8 & 109.7 & 2 & 0.27 & 41.3 & 0.67 & $64.2+11.9$ \\
\hline \multicolumn{9}{|l|}{ Strong opioids } \\
\hline Oxycodone & 76 & 6.7 & 32.3 & 2 & 0.43 & 36.0 & 0.87 & $64.4+12.8$ \\
\hline Morphine & 74 & 6.6 & $\mathrm{NA}^{*}$ & $\mathrm{NA}^{*}$ & NA & 35.1 & 0.81 & $64.2+10.8$ \\
\hline Hydromorphone & 63 & 5.6 & 6.7 & 1 & 0.33 & 36.4 & 0.86 & $65.7+11.4$ \\
\hline Methadone & 7 & 0.6 & 32.4 & 3 & 1.29 & 53.7 & 100.00 & $72.3+7.9$ \\
\hline
\end{tabular}

${ }^{*}$ Presentation most used in bottle with dropper. ${ }^{a}$ Relationship between the mean dose and the defined daily dose. Defined daily dose (DDD): gold standard measuring unit for international drug utilization, monitoring, and research (recommended by the WHO). Each medication has a daily dose defined by the WHO; the calculation presented is the ratio between the average used and the defined daily dose. Being 1, the use of the same dose recommended as DDD.

TABLE 3: Multivariate analysis to identify variables that were associated with a greater probability of receiving opioid analgesics for a period longer than 12 months in a cohort of 1,129 patients with rheumatoid arthritis in Colombia.

\begin{tabular}{lccccc}
\hline Variable & Beta & Sig $^{a}$ & $\mathrm{OR}^{b}$ & \multicolumn{2}{c}{$95 \% \mathrm{CI}^{c}$} \\
& & & & Lower & Upper \\
\hline Gender: female & -0.018 & 0.927 & 0.985 & 0.71 & 1.366 \\
Age <45 years & Ref & 0.004 & Ref & Ref & Ref \\
Age: $45-64.9$ years & 0.585 & 0.014 & 1.782 & 1.127 & 2.819 \\
Age $>65$ years & 0.813 & 0.001 & 2.235 & 1.389 & 3.598 \\
Treated in Bogotá & -0.249 & 0.113 & 0.783 & 0.579 & 1.06 \\
Treated in Manizales & 0.734 & 0.01 & 2.067 & 1.187 & 3.596 \\
Acetaminophen & 0.189 & 0.24 & 1.202 & 0.884 & 1.634 \\
Use of antidepressants & 0.446 & 0.001 & 1.553 & 1.193 & 2.021 \\
Use of anticonvulsants & 1.192 & 0.001 & 3.163 & 1.606 & 6.230 \\
Use of benzodiazepines & 1.182 & 0.004 & 3.203 & 1.462 & 7.018 \\
Treated with biological & 0.422 & 0.014 & 1.524 & 1.09 & 2.131 \\
DMARDs & & & & & \\
Use of systemic & 0.474 & 0.01 & 1.61 & 1.121 & 2.313 \\
corticosteroids & & & & & \\
\hline
\end{tabular}

$\overline{{ }^{a} \text { Significance level. }{ }^{b} \text { OR: odds ratio. }{ }^{c} 95 \% \text { confidence interval. DMARDs: }}$ disease modifying antirheumatic drugs.

The relationship found with the use of opioid analgesics for more than 12 months in the patients who were receiving therapy with biological DMARDs may be due to the fact that this group of patients shows a greater activity of the disease and therefore requires analgesic therapy. There are also reports showing that the greater and less-controlled inflammatory activity results in more pain $[16,21]$. Therefore, the association with the use of systemic corticosteroids may be related to the increased inflammatory activity in the group of patients with RA who required opioids [10, 22].

Different studies have described the relationship of RArelated pain with depression and anxiety and, therefore, the use of drugs for these clinical conditions $[10,16]$, which may explain why patients who were receiving antidepressants and benzodiazepines had a higher probability of receiving opioids for more than 12 months. It has been established that depression can increase the sensation and perception of pain, which, when correlated with the activity of RA, can increase the use of opioids [23]. Attending physicians who identify this dual condition should seek adequate, and especially safe, alternatives for effective analgesic management [18, 24-26].

The limitations of this study include those which inherent to an observational study, such as insufficient clinical records on disease activity, the time of RA evolution, or the values of acute phase reactants that could show relationships with the use of opioid analgesics. It was also not possible to determine the particular indication for the use of an opioid (measurements of pain severity are missing, so the opioid 
indication according to pain intensity could not be assessed) and whether pain originated from a condition other than the joint disease; therefore, it could not be identified if opioids may be used for conditions other than RA in this population nor was it possible to evaluate the potential adverse reactions or problems related to the medications. In addition, it was not possible to confirm opioid, NSAIDs, or acetaminophen purchases outside of the health system or drug dispensing system (NSAIDs and acetaminophen are over-the-counter medications in Colombia). The results of this study can be applied to populations of adult RA patients with similar insurance coverage, and finally, these results may not represent the current prescription patterns of opioids in RA patients. This study does have strengths such as the source of information that has been validated in different studies and the stringency of monitoring of the monthly dispensing of drugs to patients with RA, which ensures that the opioid was delivered directly to the correct person.

\section{Conclusions}

Based on the above findings, it can be concluded that opioid analgesics are frequently used in patients with chronic rheumatic pain, even for periods longer than 12 months, although there is not enough evidence to support the efficacy and safety of opioids. Therefore, prescription of opioids should be well justified and limited to situations that clearly require the drug and, hopefully, for short periods of time. A growing trend in the use of opioids in patients with RA was observed, with a higher proportion of use than in other countries; therefore, it is important to continue studying the reasons for this behavior and apply this knowledge to reducing the risk of undesirable events, especially an overdose, tolerance, and dependence.

\section{Data Availability}

The data used to support the findings of this study are available from the corresponding author upon request (https://dx.doi.org/10.17504/protocols.io.baneidbe).

\section{Ethical Approval}

The protocol was approved by the Bioethics Committee of the Universidad Tecnológica de Pereira in the category of "research without risk."

\section{Disclosure}

The results of this research work were presented at the XVII Colombian Congress of Pharmacology and at ISPOR Latin America. The manuscript was translated to English by American Journal Experts.

\section{Conflicts of Interest}

The authors declare that they have no conflicts of interest.

\section{Authors' Contributions}

MEMD, DMRV, and MMM were involved in drafting of the manuscript, statistical analysis, results, and discussion. JEMA was involved in drafting of the manuscript, critical revision of the manuscript for important intellectual content, and study supervision.

\section{Acknowledgments}

The authors would like to thank Soffy Claritza López for her work in obtaining the database.

\section{References}

[1] J. E. Machado-Alba, A. F. Ruiz, and D. A. Medina Morales, "The epidemiology of rheumatoid arthritis in a cohort of Colombian patients," Revista Colombiana de Reumatología, vol. 22, no. 3, pp. 148-152, 2015.

[2] D. L. Scott, F. Wolfe, and T. W. Huizinga, "Rheumatoid arthritis," The Lancet, vol. 376, no. 9746, pp. 1094-1108, 2010.

[3] R. L. Nahin, "Estimates of pain prevalence and severity in adults: United States, 2012," The Journal of Pain, vol. 16, no. 8, pp. 769-780, 2015.

[4] S. L. Whittle, B. L. Richards, D. M. Van Der Heijde, and R. Buchbinder, "The efficacy and safety of opioids in inflammatory arthritis: a cochrane systematic review," The Journal of Rheumatology Supplement, vol. 90, pp. 40-46, 2012.

[5] T. Heiberg, A. Finset, T. Uhlig, and T. K. Kvien, "Seven year changes in health status and priorities for improvement of health in patients with rheumatoid arthritis," Annals of the Rheumatic Diseases, vol. 64, no. 2, pp. 191-195, 2005.

[6] D. F. Weisberg, W. C. Becker, D. A. Fiellin, and C. Stannard, "Prescription opioid misuse in the United States and the United Kingdom: cautionary lessons," International Journal of Drug Policy, vol. 25, no. 6, pp. 1124-1130, 2014.

[7] Y. Álvarez and M. Farré, "Farmacología de los opioides," Monografía Opiáceos, vol. 17, no. 2, p. 21, 2005.

[8] American Pharmacists Association, Lexicomp: Drug Information Handbook, American Pharmacists Association, Washington, DC, USA, 27th edition, 2018.

[9] J. E. Machado-Alba, A. F. Ruiz, and M. E. Machado-Duque, "Adverse drug reactions associated with the use of diseasemodifying anti-rheumatic drugs in patients with rheumatoid arthritis," Revista Panamericana de Salud Pública, vol. 36, pp. 396-401, 2014.

[10] J. A. Zamora-Legoff, S. J. Achenbach, C. S. Crowson, M. L. Krause, J. M. Davis, and E. L. Matteson, "Opioid use in patients with rheumatoid arthritis 2005-2014: a populationbased comparative study," Clinical Rheumatology, vol. 35, no. 5, pp. 1137-1144, 2016.

[11] L. S. Nelson and J. Perrone, "Curbing the opioid epidemic in the United States," JAMA, vol. 308, no. 5, pp. 457-458, 2012.

[12] A. D. Furlan, J. A. Sandoval, A. Mailis-Gagnon, and E. Tunks, "Opioids for chronic noncancer pain: a meta-analysis of effectiveness and side effects," Canadian Medical Association Journal, vol. 174, no. 11, pp. 1589-1594, 2006.

[13] A. S. B. Bohnert, M. Valenstein, M. J. Bair et al., "Association between opioid prescribing patterns and opioid overdoserelated deaths," JAMA, vol. 305, no. 13, pp. 1315-1321, 2011.

[14] W. M. Compton and N. D. Volkow, "Abuse of prescription drugs and the risk of addiction," Drug and Alcohol Dependence, vol. 83, no. 1, pp. S4-S7, 2006. 
[15] B. Han, W. M. Compton, C. Blanco, E. Crane, J. Lee, and C. M. Jones, "Prescription opioid use, misuse, and use disorders in US adults: 2015 national survey on drug use and health," Annals of Internal Medicine, vol. 167, no. 5, pp. 293-301, 2017.

[16] Y. C. Lee, J. Kremer, H. Guan, J. Greenberg, and D. H. Solomon, "Chronic opioid use in rheumatoid arthritis: prevalence and predictors," Arthritis \& Rheumatology, vol. 71, no. 5, pp. 670-677, 2019.

[17] E. Kalso, J. E. Edwards, A. R. Moore, and H. J. McQuay, "Opioids in chronic non-cancer pain: systematic review of efficacy and safety," Pain, vol. 112, no. 3, pp. 372-380, 2004.

[18] T. K. Nuckols, L. Anderson, I. Popescu et al., "Opioid prescribing: a systematic review and critical appraisal of guidelines for chronic pain," Annals of Internal Medicine, vol. 160, no. 1, pp. 38-47, 2014.

[19] C. A. García, J. B. Santos Garcia, M. D. Rosario Berenguel Cook et al., "Undertreatment of pain and low use of opioids in latin America," Pain Management, vol. 8, no. 3, pp. 181-196, 2018.

[20] M. A. Ruiz Iban, J. Benavides, J. P. Forero et al., "Use of strong opioids for chronic pain in osteoarthritis: an insight into the latin American reality," Expert Review of Clinical Pharmacology, vol. 11, no. 1, pp. 47-59, 2018.

[21] K. M. Dunn, K. W. Saunders, C. M. Rutter et al., "Opioid prescriptions for chronic pain and overdose," Annals of Internal Medicine, vol. 152, no. 2, pp. 85-92, 2010.

[22] J. E. Machado-Alba, A. Gaviria-Mendoza, C. A. Vargas-Mosquera, A. F. Gil-Restrepo, and L. C. Romero-Zapata, "Opioid prescribing patterns and costs in a large group of patients in Colombia," Journal of Pain \& Palliative Care Pharmacotherapy, vol. 31, no. 1, pp. 57-65, 2017.

[23] Y. C. Lee, C. O. Bingham, R. R. Edwards et al., "Association between pain sensitization and disease activity in patients with rheumatoid arthritis: a cross-sectional study," Arthritis Care \& Research, vol. 70, no. 2, pp. 197-204, 2018.

[24] A. G. Mensah-Nyagan, L. Meyer, V. Schaeffer, C. Kibaly, and C. Patte-Mensah, "Evidence for a key role of steroids in the modulation of pain," Psychoneuroendocrinology, vol. 34, no. 1, pp. S169-S177, 2009.

[25] M. Y. Imran, E. A. Saira Khan, N. M. Ahmad, S. Farman Raja, M. A. Saeed, and I. Ijaz Haider, "Depression in rheumatoid arthritis and its relation to disease activity," Pakistan Journal of Medical Sciences, vol. 31, pp. 393-397, 2015.

[26] S. Ryan and B. McGuire, "Psychological predictors of pain severity, pain interference, depression, and anxiety in rheumatoid arthritis patients with chronic pain," British Journal of Health Psychology, vol. 21, no. 2, pp. 336-350, 2016. 